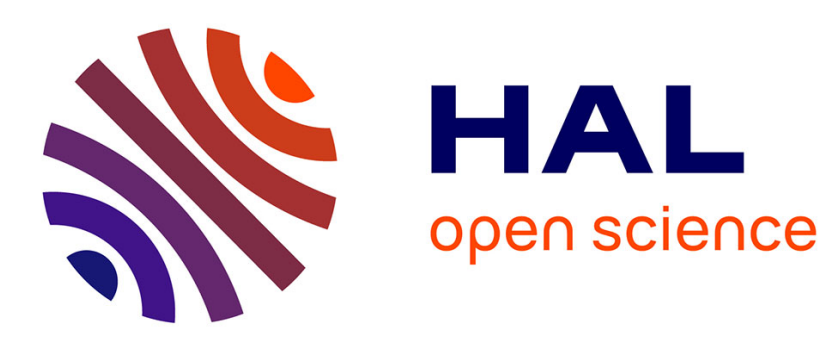

\title{
FeCl2 in a transverse magnetic field : inequivalent behaviour of the two sublattices at $0 \mathrm{~K}$
}

\author{
J.A. Nasser
}

\section{To cite this version:}

J.A. Nasser. $\mathrm{FeCl} 2$ in a transverse magnetic field : inequivalent behaviour of the two sublattices at 0 K. Journal de Physique, 1979, 40 (1), pp.51-56. 10.1051/jphys:0197900400105100 . jpa-00208884

\section{HAL Id: jpa-00208884 https://hal.science/jpa-00208884}

Submitted on 1 Jan 1979

HAL is a multi-disciplinary open access archive for the deposit and dissemination of scientific research documents, whether they are published or not. The documents may come from teaching and research institutions in France or abroad, or from public or private research centers.
L'archive ouverte pluridisciplinaire HAL, est destinée au dépôt et à la diffusion de documents scientifiques de niveau recherche, publiés ou non, émanant des établissements d'enseignement et de recherche français ou étrangers, des laboratoires publics ou privés. 


\title{
$\mathrm{FeCl}_{2}$ in a transverse magnetic field : inequivalent behaviour of the two sublattices at $0 \mathrm{~K}$
}

\author{
J. A. Nasser \\ DPh-G/PSRM, CEN Saclay, B.P. No 2, 91190 Gif sur Yvette, France
}

(Reçu le 3 janvier 1978, révisé le 10 juillet 1978, accepté le 2 octobre 1978)

\begin{abstract}
Résumé. - Nous étudions à $0 \mathrm{~K}$ dans $\mathrm{FeCl}_{2}$ - un antiferromagnétique uniaxe pour $T \leqslant T_{\mathrm{N}}$ - les effets élastiques et magnétiques dus au couplage spin-réseau introduit par le champ cristallin de l'ion $\mathrm{Fe}^{2+}$. A $0 \mathrm{~K}$, en champ perpendiculaire à l'axe de facile aimantation, qui coincide avec l'axe $\overline{3}$ du groupe ponctuel $(\overline{3} \mathrm{~m})$ des ions fer, les résultats sont les suivants : il s'établit des déformations qui détruisent la symétrie du cristal. Il apparaît une aimantation globale qui a une composante suivant la direction du champ mais aussi une composante suivant l'axe ternaire; ce dernier résultat bien qu'a priori surprenant est conforme aux considérations de symétrie. Par ailleurs les moments des deux sous-réseaux ne sont plus contenus dans le plan de l'axe et du champ, et ne s'inclinent pas également en direction du champ. Enfin, pour un champ de module fixé, les résultats varient avec la direction du champ dans le plan perpendiculaire à l'axe ternaire.
\end{abstract}

\begin{abstract}
For the uniaxial antiferromagnet $\mathrm{FeCl}_{2}$, we study the elastic and magnetic effects at $0 \mathrm{~K}$ due to the magneto-elastic coupling introduced by the crystal field of the $\mathrm{Fe}^{2+}$ ions. In a magnetic field $\mathrm{H}$ perpendicular to the easy magnetization axis, which is the $\overline{3}$ axis of the point group $(\overline{3} \mathrm{~m})$ of $\mathrm{Fe}^{2+}$ ions, strains occur destroying the crystal symmetry and a bulk magnetization appears with components along the field direction and also along the ternary axis. The presence of the component along the ternary axis is compatible with symmetry considerations. Also each of the magnetic moments of the two sublattices no longer lies in the plane defined by $\mathbf{H}$ and the ternary axis and they do not equally tilt towards the field direction. For $\mathbf{H}$ of a given size, the results vary quantitatively with the direction of $\mathbf{H}$ in the plane perpendicular to the ternary axis.
\end{abstract}

1. Introduction. - The static magnetic properties [1] of $\mathrm{FeCl}_{2}$ - an uniaxial antiferromagnet - as well as its magnetic excitations [2] have been extensively studied. But whereas some experiments [3] have clearly shown the importance of the magneto-elastic coupling in this compound, a number of static experimental results obtained either in zero field or with the field parallel to the easy magnetization axis, can be satisfactorily interpreted with a model due to Ôno [4], which neglects the crystal strains. This arises as under these experimental conditions the magneto-elastic coupling does not qualitatively modify the results of the model. In a transverse field, however, the minimization of the free energy can take place through the introduction of strains which destroy the crystal symmetry giving a magnetic structure which cannot be predicted within Ôno's model. For this structure the two sublattices show inequivalent behaviour in the antiferromagnetic phase.

One aspect of this inequivalent behaviour has already been observed in the Mössbauer spectrum of the $\mathrm{Fe}^{2+}$ ion [5]. The splitting of the right hand absorption line into two components as shown on figure 1 , which shows a $\cos 3 \varphi$ dependence, is due to the fact that the projection on the field direction of the magnetic moment of one sublattice is not equal to that of the other sublattice.

The above mentioned magnetic and elastic effects arise from the variations of crystalline field of $\mathrm{Fe}^{2+}$ with crystal strains. These variations are significative only if the orbital moment is not completely quenched as in the case for $\mathrm{FeCl}_{2}$. The variations disturb the orbital state of $\mathrm{Fe}^{2+}$ which, because of spin-orbit coupling, modifies its spin state.

If $\varepsilon_{p q}$ and $L_{i}(p, q, i=1,2,3)$ are the respective components, in an orthogonal frame $\left\{\mathrm{O} x_{i}\right\}$, of the strain tensor and the orbital moment of the free $\mathrm{Fe}^{2+}$ ion, the development in $\varepsilon_{p q}$ of the crystalline field can be written $\sum_{p, q, i, j} A_{p q i j} \varepsilon_{p q}\left(L_{i} L_{j}+L_{j} L_{i}\right)$. The non-zero components of the tensor $A_{p q i j}$ are determined from the point group $(\overline{3} \mathrm{~m})$ of the $\mathrm{Fe}^{2+}$ ions in the zero field paramagnetic phase. If one considers the $\varepsilon_{p q}$ as variational parameters, the development in $\varepsilon_{p q}$ is an 


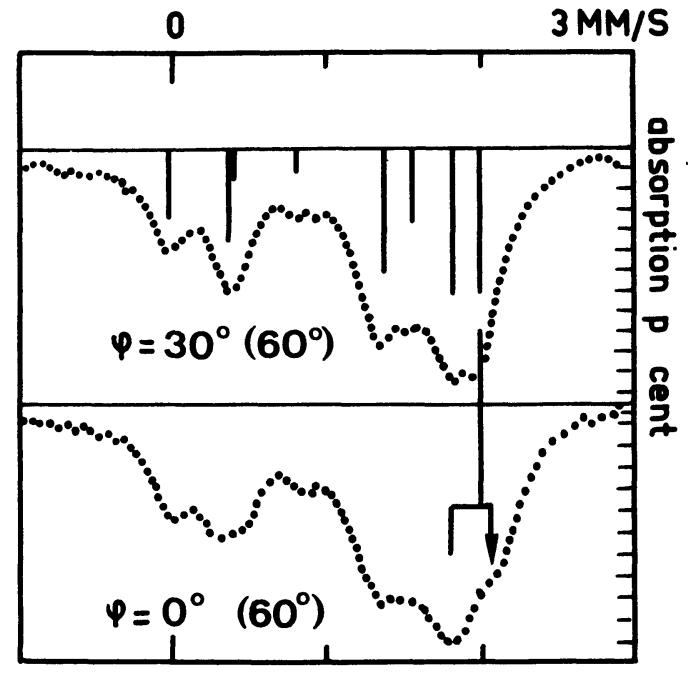

1 Applied Field in $\mathrm{Fe} \mathrm{Cl}_{2}$

Fig. 1. - Mössbauer spectrum of $\mathrm{Fe}^{2+}$ at $4.2 \mathrm{~K}$ and $H=60 \mathrm{kOe}$; $\varphi$ is the angle defined in figure 2.

operator which can be decomposed into two parts, one having $\overline{3} \mathrm{~m}$ symmetry, and the other, $\delta w$, with a lower symmetry. $\delta w$ is certainly smaller than the operator of trigonal symmetry and also smaller than the spin-lattice coupling operator due to the exchange interaction. Nevertheless it is $\delta w$ which is responsible for the effects studied here. The operator with $\overline{3} \mathrm{~m}$ symmetry (as well as the development in $\varepsilon_{p q}$ of exchange integrals) can cause the appearance of strains which however cannot lower the crystal symmetry and can lead to the appearance of magnetic effects qualitatively similar to those contained in Ôno's model.

The importance in $\mathrm{FeCl}_{2}$ of spin-lattice coupling associated with the strain variation of crystal field parameters was initially described by G. Laurence and D. Petitgrand [6] following Laurence's thermal conductivity measurements in $\mathrm{FeCl}_{2}$ [2] (G. Laurence 1971). Subsequent theoretical [7] and experimental [8] studies have examined the consequences of this spinlattice coupling on the magnetic excitations in $\mathrm{FeCl}_{2}$. We predict here the influence of this coupling on the static properties of $\mathrm{FeCl}_{2}$.

In this study we consider $\delta w$ to be a perturbation to be added to Ôno's model at $0 \mathrm{~K}$. One can verify that our results agree with symmetry considerations.

2. $\delta w$ as a perturbation of the rigid crystal model. Figure 2 represents the antiferromagnetic structure of $\mathrm{FeCl}_{2}$ at $0 \mathrm{~K}$ in zero field. The easy magnetization axis is the ternary axis. In the $\mathrm{O} x y z$ frame, $\mathrm{Oz}$ is parallel to the ternary axis and $O x$ is contained in a mirror plane.

In Ôno's model this structure is explained by taking into account crystalline field, spin-orbit coupling, ferromagnetic exchange between the $\mathrm{Fe}^{2+}$ ions contained in the same plane perpendicular to the ternary axis and the antiferromagnetic exchange between

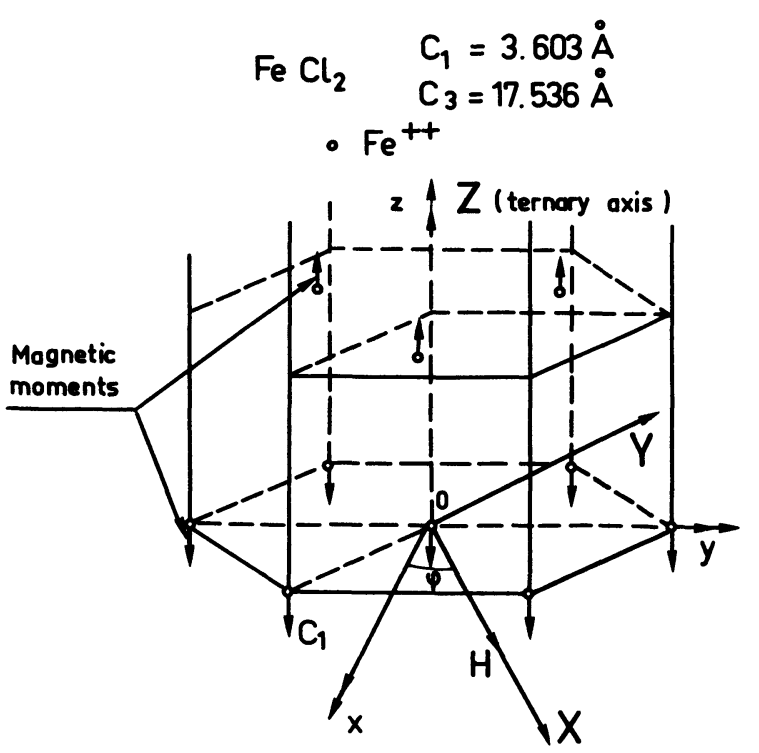

Fig. 2. - Antiferromagnetic order of $\mathrm{FeCl}_{2}$ at $0 \mathrm{~K}$ and $\mathbf{H}=0$.

the $\mathrm{Fe}^{2+}$ ions contained in two successive planes perpendicular to the ternary axis. Starting from the fundamental level of the free $\mathrm{Fe}^{2+}$ ion $(L=2, S=2)$, these interactions are treated as successively decreasing perturbations.

After diagonalizing the crystalline field and the spin-orbit coupling, one can consider the fundamental level of each ion as a triplet in which there exists an axial anisotropy term. If one introduces a fictitious spin $\mathbf{s}, s=1$, the anisotropy term can be written $-D\left(s_{z}^{2}-1\right)$.

If one uses a two-sublattice molecular field approximation and taking into account $\delta w$ and the Zeeman effect in a transverse field, the fundamental level of each ion can be obtained by diagonalizing the Hamiltonian $\mathcal{H}_{\alpha}^{\prime}$ within the triplet, where :

$\mathcal{H}_{\alpha}^{\prime}=\mathscr{H}_{\alpha}+\delta w$.

$\mathcal{H}_{\alpha}$, the Hamiltonian used by Ôno, can be written :

$$
\begin{aligned}
\mathscr{H}_{\alpha}= & -D\left(s_{z}^{2}-1\right)-s_{x}\left(J_{1 \perp} x_{\alpha}^{\prime}+J_{2 \perp} x_{\beta}^{\prime}\right)- \\
& -s_{y}\left(J_{1 \perp} y_{\alpha}^{\prime}+J_{2 \perp} y_{\beta}^{\prime}\right)-s_{z}\left(J_{1 \|} z_{\alpha}^{\prime}+J_{2 \|} z_{\beta}^{\prime}\right) \\
& +g_{\perp} \mu_{\mathrm{B}} H\left(s_{x} \cos \varphi+s_{y} \sin \varphi\right)
\end{aligned}
$$

with $\alpha=1,2$ and $\beta=2,1 ; 1$ and 2 are the indices of the two sublattices. In this expression : $s_{x}, s_{y}, s_{z}$ are the components of $\mathbf{s}$ in $\mathrm{Ox}, \mathrm{Oy}, \mathrm{Oz} . x_{\alpha}^{\prime}, y_{\alpha}^{\prime}, z_{\alpha}^{\prime}$ are the mean values of $s_{x}, s_{y}, s_{z}$ in the sublattice $\alpha$; they are the solutions of the molecular field equations. $J_{1 \|}$ and $J_{1 \perp}$ characterize the ferromagnetic interaction. $H$ is the modulus of the transverse field $\mathbf{H}$; $(\mathrm{Ox}, \mathbf{H})=\varphi . \mu_{\mathrm{B}}$ is the Bohr magneton and $g_{\perp}$ a spectroscopic term $\left(g_{\perp}=3.22\right)$.

$\delta w$ can be written in terms of the components $L_{x}$, $L_{y}, L_{z}$ of the free $\mathrm{Fe}^{2+}$ ion orbital momentum $\mathbf{L}$ :

$$
\begin{aligned}
\delta w= & \varepsilon_{13}\left(K_{1} R+K_{1}^{\prime} U\right)+\varepsilon_{23}\left(K_{1} S-K_{1}^{\prime} V\right)+ \\
& +\left(\varepsilon_{11}-\varepsilon_{22}\right)\left(K_{2} U-K_{2}^{\prime} R\right)+2 \varepsilon_{12}\left(K_{2} V+K_{2}^{\prime} S\right)
\end{aligned}
$$


where $K_{1}, K_{1}^{\prime}, K_{2}, K_{2}^{\prime}$ are real constants, and where

$$
\begin{array}{ll}
R=L_{x} L_{z}+L_{z} L_{x}, & U=L_{x}^{2}-L_{y}^{2} \\
S=L_{y} L_{z}+L_{z} L_{y}, & V=L_{x} L_{y}+L_{y} L_{x} .
\end{array}
$$

The expression for $\delta w$ is limited to second order in $L_{x}, L_{y}, L_{z}$.

If $\delta w$ is treated as a perturbation compared with $\mathscr{H}_{\alpha}, x_{\alpha}^{\prime}, y_{\alpha}^{\prime}$ and $z_{\alpha}^{\prime}$ can be written :

$x_{\alpha}^{\prime}=x_{\alpha}+\delta x_{\alpha}, \quad y_{\alpha}^{\prime}=y_{\alpha}+\delta y_{\alpha}, \quad z_{\alpha}^{\prime}=z_{\alpha}+\delta z_{\alpha}$

where $\delta x_{\alpha}, \delta y_{\alpha}$ and $\delta z_{\alpha}$ are zero if $\delta w$ is neglected.

2.1 Results Neglecting $\delta w$. - For $H$ smaller than the critical field $H_{\mathrm{c}}(\simeq 130 \mathrm{kOe})$ of the antiferromagnetic $\rightarrow$ saturated paramagnetic transition, the compound is in an antiferromagnetic phase. The magnetic moments of the two sublattices are confined in the plane defined by $\mathrm{Oz}$ and $\mathrm{H}$ and are equally inclined with respect to the field. Furthermore the projection of each sublattice moment on the field direction is independent of $\varphi$. This situation is described by the following relations :

$z_{1}=-z_{2}=z, \quad x_{1}=x_{2}=x \cos \varphi$,

$y_{1}=y_{2}=x \sin \varphi$

$z$ and $x$ being only dependent on $H$; we choose $z \geqslant 0$. We can finally prove the following results :

- $H_{1}$ and $H_{2}$ have the same eigenvalues which depend only upon $H$. We call them $E_{k}$ with $k=\mathrm{I}$, II, III in increasing order.

- The normalized eigenvectors $\left|\psi_{k}^{1}\right\rangle$ associated with the eigenvalue $E_{k}$ of $\mathscr{H}_{1}$ can be written :

$\left|\psi_{k}^{1}\right\rangle=\lambda_{k} \mathrm{e}^{i \varphi}|-1\rangle+\mu_{k}|0\rangle+v_{k} \mathrm{e}^{-i \varphi}|1\rangle$,

where $\lambda_{k}, \mu_{k}$ and $v_{k}$ are real and where $\left|m_{s}= \pm 1,0\right\rangle$ are the eigenvectors of $s_{z}$ and $\mathbf{s}^{2}$. We obtain $\left|\psi_{k}^{2}\right\rangle$ by permuting $\lambda_{k}$ and $v_{k}$.

- The equations of the molecular field can be written :

$z=\left\langle\psi_{\mathrm{I}}^{1}\left|s_{z}\right| \psi_{\mathrm{I}}^{1}\right\rangle=v_{\mathrm{I}}^{2}-\lambda_{\mathrm{I}}^{2}$

$x \cos \varphi=\left\langle\psi_{\mathrm{I}}^{1}\left|s_{x}\right| \psi_{\mathrm{I}}^{1}\right\rangle=\sqrt{2} \mu_{\mathrm{I}}\left(\lambda_{\mathrm{I}}+v_{\mathrm{I}}\right) \cos \varphi$.
The fundamental energy $\left(E_{1}\right)_{\alpha}$ of an ion of sublattice $\alpha$ is defined by :

$\left(E_{\mathrm{l}}\right)_{\alpha}=\left\langle\psi_{\mathrm{I}}^{\alpha}\left|\mathscr{H}_{\alpha}\right| \psi_{\mathrm{I}}^{\alpha}\right\rangle$

$E$ which is the total energy per unit volume depends only on $\mathbf{H}$.

We have numerically calculated the values of $x$ and $z$ for different values of the magnetic field between 0 and $160 \mathrm{kOe}$. The results are reported in table I. They depend on the values of the set of parameters $\left\{D, J_{1 \perp}, J_{1 \|}, J_{2 \perp}, J_{2 \|}\right\}$ contained in $\mathcal{H}_{\alpha}$. This set of parameters was determined for the first time by Ôno et al. [4] using a set of experimental results. Subsequently a number of modifications of these parameters have been proposed (see Alben [2] (1969), Carrara [1] (1968), Fert [1] (1973), etc...). It is not the purpose of this article to compare the values proposed by the different authors. We use here the values of Ôno, namely

$D=12.7 \mathrm{~cm}^{-1}$

$J_{1 \perp}=13.08 \mathrm{~cm}^{-1}, \quad J_{1 \|}=18.32 \mathrm{~cm}^{-1}$

$J_{2 \perp}=-1.45 \mathrm{~cm}^{-1}, \quad J_{2 \|}=-2.03 \mathrm{~cm}^{-1}$.

One can see, in table $\mathrm{I}$, that in the antiferromagnetic phase, $x$ is not a linear function of the field. We thus confirm the numerical results obtained by Gelard et al. [1]. These authors have shown that non-linearity of $x$ can partially explain the non linear experimental behaviour of the bulk aimantation of $\mathrm{FeCl}_{2}$ in a transverse magnetic field (Carrara [1](1969)).

2. 2 Results TAKING INTO ACCOUNT $\delta w$. - Using first order perturbation theory, the energy of one ion of the sublattice $\alpha$ can be written :

$\left(E_{\mathrm{I}}\right)_{\alpha}+(\delta E)_{\alpha}=\left\langle\psi_{\mathrm{I}}^{\alpha}\left|\mathcal{H}_{\alpha}^{\prime}\right| \psi_{\mathrm{I}}^{\alpha}\right\rangle$

and $E+\delta E\left(\varepsilon_{p q}, \mathbf{H}\right)$ which is the energy per unit volume can be found. $\delta E$ depends on the strains and on $\mathbf{H}$. If $H=0, \lambda_{\mathrm{I}}=0$ and $\mu_{\mathrm{I}}=0$, it follows that $(\delta E)_{\alpha}$ and $\delta E$ are zero, and that the effects described here no longer exist.

To first order the fundamental state of an ion of the $\alpha$ sublattice $\left|\phi_{\alpha}\right\rangle$ is given by :

$\left|\phi_{\alpha}\right\rangle=\left|\psi_{\mathrm{I}}^{\alpha}\right\rangle+p_{\alpha}\left|\psi_{\mathrm{II}}^{\alpha}\right\rangle+q_{\alpha}\left|\psi_{\mathrm{III}}^{\alpha}\right\rangle$.

\begin{tabular}{|c|c|c|c|c|c|c|c|c|c|c|c|}
\hline$H(\mathrm{kOe})$ & 0 & 1 & 10 & 20 & 40 & 60 & 80 & 100 & 120 & 140 & 160 \\
\hline$z$ & 1.00 & 1.00 & $9.98 \times 10^{-1}$ & $9.90 \times 10^{-1}$ & $9.58 \times 10^{-1}$ & $9.00 \times 10^{-1}$ & $8.02 \times 10^{-1}$ & $6.41 \times 10^{-1}$ & $3.18 \times 10^{-1}$ & 0.00 & 0.00 \\
\hline$x$ & 0.00 & $-7.01 \times 10^{-3}$ & $-7.02 \times 10^{-2}$ & $-1.41 \times 10^{-1}$ & $-2.85 \times 10^{-1}$ & $-4.34 \times 10^{-1}$ & $-5.90 \times 10^{-1}$ & $-7.55 \times 10^{-1}$ & $-9.29 \times 10^{-1}$ & $-9.82 \times 10^{-1}$ & $-9.85 \times 10^{-1}$ \\
\hline$Q$ & 0.00 & $-3.40 \times 10^{-6}$ & $-3.38 \times 10^{-4}$ & $-1.35 \times 10^{-3}$ & $-5.40 \times 10^{-3}$ & $-1.21 \times 10^{-2} \mid$ & $-2.15 \times 10^{-2}$ & $-3.35 \times 10^{-2}$ & $-4.80 \times 10^{-2}$ & $-1.23 \times 10^{-1}$ & $-1.22 \times 10^{-1}$ \\
\hline$\left(Q M_{X}^{+}\right) \mathrm{cm}$ & 0.00 & $<10^{-7}$ & $8.00 \times 10^{-7}$ & $6.1 \times 10^{-6}$ & $4.83 \times 10^{-5}$ & $1.61 \times 10^{-4}$ & $3.75 \times 10^{-4}$ & $7.17 \times 10^{-4}$ & $1.21 \times 10^{-3}$ & $-1.50 \times 10^{-4}$ & $-1.25 \times 10^{-4}$ \\
\hline$\left(Q M_{Z}^{+}\right) \mathrm{cm}$ & 0.00 & $<10^{-7}$ & $-3.00 \times 10^{-7}$ & $-2.2 \times 10^{-6}$ & $-1.57 \times 10^{-5}$ & $-4.02 \times 10^{-5}$ & $-3.41 \times 10^{-5}$ & $1.82 \times 10^{-4}$ & $1.40 \times 10^{-3}$ & $3.65 \times 10^{-3}$ & $2.51 \times 10^{-3}$ \\
\hline$(Q M$ & 0.00 & $<10^{-7}$ & $-4.00 \times 10^{-6}$ & $-1.55 \times 10^{-5}$ & $-5.36 \times 10^{-5}$ & $-8.65 \times 10^{-5}$ & $-5.82 \times 10^{-5}$ & $1.22 \times 10^{-4}$ & $4.23 \times 10^{-4}$ & 0.00 & 0.00 \\
\hline$(Q M$ & 0.00 & $<10^{-7}$ & $-4.00 \times 10^{-6}$ & $-1.55 \times 10^{-5}$ & $-6.12 \times 10^{-5}$ & $-1.28 \times 10^{-4}$ & $-2.00 \times 10^{-4}$ & $-2.45 \times 10^{-4}$ & $-1.72 \times 10^{-4}$ & 0.00 & 0.00 \\
\hline$\left(Q M_{Z}^{-}\right.$ & & $10^{-7}$ & $1.00 \times 10^{-7}$ & $1.00 \times 10^{-6}$ & $1.71 \times 10^{-5}$ & $9.20 \times 10^{-5}$ & $3.24 \times 10^{-4}$ & $9.82 \times 10^{-4}$ & $4.07 \times 10^{-3}$ & 0.00 & 0.00 \\
\hline
\end{tabular}

Table I. 
We deduce the molecular field equations for $x_{\alpha}^{\prime}, y_{\alpha}^{\prime}$ and $z_{\alpha}^{\prime}$ :

$x_{\alpha}^{\prime}=\left\langle\phi_{\alpha}\left|s_{x}\right| \phi_{\alpha}\right\rangle=x \cos \varphi+\delta x_{\alpha}$

$y_{\alpha}^{\prime}=\left\langle\phi_{\alpha}\left|s_{y}\right| \phi_{\alpha}\right\rangle=x \sin \varphi+\delta y_{\alpha}$

$z_{\alpha}^{\prime}=\left\langle\phi_{\alpha}\left|s_{z}\right| \phi_{\alpha}\right\rangle=\varepsilon_{\alpha} z+\delta z_{\alpha}$

with $\varepsilon_{1}=1$ and $\varepsilon_{2}=-1$.

By solving these equations we can express $\delta x_{\alpha}, \delta y_{\alpha}$ and $\delta z_{\alpha}$ in terms of the strains $\varepsilon_{p q}$.

The strains at $0 \mathrm{~K}$ are obtained by minimizing with respect to $\varepsilon_{p q}$, the total energy which contains the magnetic energy $E(\mathbf{H})$, the magneto-elastic energy $\delta E\left(\mathbf{H}, \varepsilon_{p q}\right)$ and the elastic energy

$\sum_{p q p^{\prime} q^{\prime}} \frac{1}{2} C_{p q p^{\prime} q^{\prime}} \varepsilon_{p q} \varepsilon_{p^{\prime} q^{\prime}}$

The magnetic structure at $0 \mathrm{~K}$ can be obtained by transferring these values into the molecular field solutions $\left(1^{\prime}\right)$.

The numerical values of the results presented below depend on the values of the constants $K_{1}, K_{1}^{\prime}, K_{2}, K_{2}^{\prime}$ and of the set of parameters $\left\{D_{1}, J_{1 \perp}, J_{1 \|}, J_{2 \perp}, J_{2 \|}\right\}$ contained in $\mathcal{H}_{\alpha}$.

3. Results in a frame linked to the magnetic field. We consider a frame $O X Y Z$ such that $O X$ is parallel to $\mathrm{H}$ and $\mathrm{OZ}$ parallel to $\mathrm{Oz} . \varepsilon_{p q}^{\prime}$ are strain tensor components in this frame and $m_{X \alpha}, m_{Y \alpha}, m_{Z_{\alpha}}$ are the magnetic moment components of the sublattice in units of $-g_{\perp} \mu_{\mathrm{B}}$.

3.1 Strain. - For the strain the following relations exist :

$$
\begin{aligned}
\varepsilon_{11}^{\prime}-\varepsilon_{22}^{\prime} & =\frac{N Q}{\Delta_{1}}\left[C_{15} A+C_{55} \frac{B \sqrt{2}}{2}\right] \\
2 \varepsilon_{12}^{\prime} & =0 \\
\varepsilon_{13}^{\prime} & =-\frac{N Q}{\Delta_{1}}\left[C_{66} \frac{A}{2}+C_{15} \frac{B \sqrt{2}}{4}\right] \cos 3 \varphi \\
\varepsilon_{23}^{\prime} & =\frac{N Q}{\Delta_{1}}\left[C_{66} \frac{A}{2}+C_{15} \frac{B \sqrt{2}}{4}\right] \sin 3 \varphi
\end{aligned}
$$

with

$\Delta_{1}=2\left(C_{66} C_{55}-C_{15}^{2}\right), \quad A=K_{1}-\frac{\sqrt{2}}{4} K_{1}^{\prime}$

and

$B=\frac{\sqrt{2}}{4} K_{2}+K_{2}^{\prime}$.

In these expressions, $N$ is the number of $\mathrm{Fe}^{2+}$ ions per unit volume, $C_{p q}(p, q=1$ to 6$)$ are the elastic constants, in matrix notation [9], relative to the frame Oxyz. $Q$ which depends only on $H$ is well represented by a function in $H^{2}$ and the values are given in table $\mathrm{I}$.
We can verify that these results respect the symmetry considerations : when the strains are expressed in the frame $\mathrm{O} x y z$ we see that they are even functions of $\mathbf{H}$. The first term of the expansion of these functions in $\mathbf{H}$ is $\varepsilon_{p q}=t_{p q r s} H_{r} H_{s}(p, q=1,2,3$ and $r, s=1,2$ with $1 \rightarrow \mathrm{Ox}, 2 \rightarrow \mathrm{Oy}, 3 \rightarrow \mathrm{Oz}) ; t_{\text {pqrs }}$ is invariant in the symmetry operations $\overline{3} \mathrm{~m}$. By examining the tensor $t_{p q r s}$ we find that its non zero components verify the following relations :

$t_{1111}=t_{2222} ; \quad t_{1122}=t_{2211} ;$

$2 t_{1221}=t_{1111}-t_{1122}$;

$t_{1311}=-t_{1322} ; t_{1311}=-t_{2312}$.

It is easy to show that the equations (2) follow from these relations.

3.2 Magnetic STRUCtURe (IN UNITS $-g_{\perp} \mu_{\mathrm{B}}$ ). i) For the total magnetization we find :

$m_{X 1}+m_{X 2}=2 x-\frac{N W}{\Delta_{1}}\left(M_{X}^{+} Q\right)$

$m_{Y 1}+m_{Y 2}=0$

$m_{Z 1}+m_{Z 2}=-\frac{N W^{\prime}}{\Delta_{1}}\left(M_{Z}^{+} Q\right) \cos 3 \varphi$

with

$$
\begin{aligned}
& W=C_{66} \frac{A^{2}}{2}+C_{15} \frac{\sqrt{2}}{2} A B+C_{55} \frac{B^{2}}{4} \\
& W^{\prime}=C_{66} \frac{A C}{2}-C_{15}\left(A D-\frac{\sqrt{2}}{4} B C\right)-C_{55} \frac{\sqrt{2}}{2} B D \\
& C=K_{1}+2 \sqrt{2} K_{1}^{\prime}, \quad D=2 \sqrt{2} K_{2}-K_{2}^{\prime} .
\end{aligned}
$$

In these expressions $x,\left(M_{X}^{+} Q\right),\left(M_{Z}^{+} Q\right)$ depend only on $H$. In table I we give their variations as a function of $H$. This table shows that for $H \leqslant 40 \mathrm{kOe} x$ is well represented by a linear function of $H$ whereas $\left(M_{X}^{+} Q\right)$ and $\left(M_{Z}^{+} Q\right)$ are well represented by cubic functions of $H$.

The relation (3.1) shows that even for $H \leqslant 40 \mathrm{kOe}$ the magnetization parallel to the field does not vary linearly with $H$. This behaviour could partially explain the experimental results obtained by Carrara [1](1969). (See also J. Gelard et al. [1] (1974)). The relation (3.3) shows that a transverse field gives rise to a magnetization along the $\mathrm{OZ}$ axis which changes sign between 80 and $100 \mathrm{kOe}$.

Again we can verify that these results respect the symmetry considerations. Relations (3) show that the magnetization components are odd functions of $\mathbf{H}$. The beginning of the development of these functions in $\mathbf{H}$ is $M_{p}=\chi_{p r} H_{r}+\chi_{p r s u} H_{r} H_{s} H_{u}(p=1,2,3$ and $r, s, u=1,2$ ), where $M_{p}$ represents the projection of the total magnetization along the $O x_{p}$ axis. The tensor $\chi_{p r}$ and $\chi_{\text {prsu }}$ are invariant in the symmetry operations $3 \mathrm{~m}$. The tensor $\chi_{p r}$ is diagonal in the frame 
Oxyz whereas the non zero components of the tensor $\chi_{p r s u}$ verify the following relations

$\chi_{1111}=\chi_{2222} ; \quad \chi_{1122}=\chi_{2112} ;$

$\chi_{1111}=3 \chi_{1122} ; \chi_{3111}=-\chi_{3122}$.

It is again easy to show that the eq. (3) follow from these relations.

ii) Difference between the magnetizations of the two sublattices.

We obtain the following relations :

$m_{X 1}-m_{X 2}=-\frac{N}{\Delta_{1}} W^{\prime}\left(M_{X}^{-} Q\right) \cos 3 \varphi$

$m_{Y 1}-m_{Y 2}=\frac{N}{\Delta_{1}} W^{\prime}\left(M_{Y}^{-} Q\right) \sin 3 \varphi$

$m_{Z 1}-m_{Z 2}=2 z-\frac{N}{\Delta_{1}} W\left(M_{Z}^{-} Q\right)$.

In these expressions $\left(M_{X}^{-} Q\right),\left(M_{Y}^{-} Q\right)$ and $\left(M_{\bar{Z}}^{-} Q\right)$ depend only on $H$ and the values are given in table $\mathrm{I}$. These expressions are zero in the saturated paramagnetic phase.

The relations (4.1), (4.2) associated with relation (3.2) show the existence of a magnetic structure which is quite new : the magnetic moments of the two sublattices point out of the plane defined by $\mathrm{O} z$ and $\mathrm{H}$ and also the moments are not equally tilted towards the field direction.

4. Conclusion. - In this study we point out one aspect of the magneto-elastic coupling associated with the influence of the crystalline field on the magnetic ion. For $\mathrm{FeCl}_{2}$ experimental measurements (by X-rays, neutrons, etc...) are necessary in order to obtain the values of the constants $A, B, C, D$ which are the only unknowns in the problem as the elastic constants are known [10].

According to a preliminary fitting of the Mössbauer spectra [5] shown in figure 1 , the difference in magne- tization of the two sublattices in the field direction is estimated to be approximately $0.1 \mu_{\mathrm{B}}$. We deduce from this result - by using table $I$ and relations (4.1), (4.2) and (3.3) - :1) that the total magnetization along the ternary axis direction is approximately $0.1 \mu_{\mathrm{B}}$ for a pair of $\mathrm{Fe}^{2+}$ ions belonging to the two sublattices ; 2) that the magnetization in the direction perpendicular to the plane defined by the field and the ternary axis is approximately $0.1 \mu_{\mathrm{B}}$ per ion. In an other way - namely by taking $N, \Delta_{1}[10]$ and $\left(M_{X}^{-} Q\right)$ equal respectively to $1.5 \times 10^{22} \mathrm{at} . / \mathrm{cm}^{3}$,

$$
9.5 \times 10^{21}\left(\mathrm{erg} / \mathrm{cm}^{3}\right) \text { and } 8.7 \times 10^{-5} \mathrm{~cm}
$$

one can deduce from the Mössbauer result and from relation (4.1) that $W^{\prime}$ is of the order of $10^{34}$. As the elastic constants are of the order of $10^{11} \mathrm{erg} / \mathrm{cm}^{3}$ [10] one can deduce that the parameters $A, B, C$ and $D$ are approximately of the order of $10^{3} \mathrm{~cm}^{-1}$. From this result and using table I and relation (2) one deduces that the strains which destroy crystal symmetry are of the order of $10^{-4}$ for a $60 \mathrm{kOe}$ field.

It would be interesting to experimentally observe results specifically relating to $\delta w$, namely those described by relations (2), (3.3), (4.1) and (4.2). The above calculations indicate that they can be observed with a strong enough field. However if strains are mechanically created, the magnetic effects should become visible in lower applied fields. But it is not possible to make a quantitative assessment of this influence of strains as the values of the parameters $A$, $B, C$ and $D$ are not known with sufficient accuracy.

It would be interesting to study these aspects of the magneto-elastic coupling in other compounds where the orbital moment of the magnetic ion is not completely quenched.

Acknowledgments. - I would like to thank C. Pailloud, J. A. Hodges, R. Bidaux and L. de Seze for comments on the manuscript and N. Boccara for very valuable suggestions concerning symmetry considerations.

\section{References}

[1] Ericson, M., de Gennes, P. G., Herpin, A., Jacrot, B., Mériel, P., J. Physique Radium 19 (1958) 617 ;

Jacobs, I. S., Lawrence, P. E., Phys. Rev. 164 (1967) 866 (see Ref.);

Carrara, P., Thesis, Paris (1968);

Carrara, P., de Gunzbourg, J., Allain, Y., J. Appl. Phys. 40 (1969) 1035 ;

lanusse, M. C., Carrara, P., Fert, A. R., Mischler, G., Redoulès, J. P., J. Physique 33 (1972) 429.

Fert, A., Thesis, Toulouse (1973);

Gelard, J., Carrara, P., Fert, A. R., J. Physique 35 (1974) 163 ;

Bertrand, Y., Fert, A. R., Gelard, J., J. Physique 35 (1974) 385 ;

Birgeneau, R. J., Shirane, G., Blume, M., Koehler, W. C., Phys. Rev. Lett. 33 (1974) 1098 ;
Fert, A. R., Gelard, J., Carrara, P., J. Phys. Chem. Solids 35 (1974) 261.

[2] Jacobs, I. S., Roberts, S., Lawrence, P. E., J. Appl. Phys. 36 (1965) 1197 ;

Alben, R., J. Phys. Soc. Japan 26 (1969) 261 ;

Bizette, H., Terrier, C., Billerey, D., Picard, J., C. R. Hebd. Séan. Acad. Sci. B 270 (1970) 545 ;

Laurence, G., Phys. Lett. A 34 (1971) 308 ;

Birgeneau, R. J., Yelon, W. B., Cohen, E., Makovsky, J., Phys. Rev. B 5 (1972) 2607;

Yelon, W. B., Birgeneau, R. J., Phys. Rev. B 5 (1972) 2615 ;

Fert, A. R., Leotin, J., Ousset, J. C., Bertrand, D., Carrara, P., Askenazy, S., Solid State Commun. 18 (1976) 327

Petitgrand, D., Meyer, P., J. Physique 37 (1976) 1417.

[3] Narath, A., Schirber, J. E., J. Appl. Phys. 37 (1966) 1124 ; 
Vettier, C., Alberts, H. L., Beille, J., Bloch, D., Intern. Conf. Magnetism, Moscow (1973) Proc. III, 501 ;

Hamman, J., Nasser, J. A., Phys. Status Solidi (b) 56 (1973) 95 ;

Nasser, J. A., J. Phys. Chem. Solids 34 (1973) 2247 ;

Nasser, J. A., Varret, F., Solid State Commun. 13 (1973) 381 ;

Vettier, C., Alber Ts, H. L., Bloch, D., Phys. Rev. Lett. 31 (1973) 1414

Johnson, K. C., Sievers, A. J., Phys. Rev. B 7 (1973) 1081 ;

Vettier, C., Yelon, W. B., J. Phys. Chem. Solids 36 (1975) 401 ;

Vettier, C., Yelon, W. B., Phys. Rev. B 11 (1975) 4700.

[4] Ôno, K., Ito, A., Fujita, T., J. Phys. Soc. Japan 19 (1964) 2119.
[5] Nasser, J. A., VarRet, F., 20th Annual Conf. M.M.M. (1974), San Francisco.

[6] Laurence, G., Petitgrand, D., Phys. Rev. B 8 (1973) 2130

[7] Lovesey, S. W., J. Phys. C 7 (1974) 2049 ;

Cracknell, A. P., J. Phys. C 7 (1974) 4323 ;

Yelon, W. B., Scherm, R., Vettier, C., Solid State Commun. 15 (1974) 391 ;

Lovesey, S. W., Comments Solid State Phys. 7 (1976) 117.

[8] Ziebeck, K. R. A., Houman, J. G., Proceedings of the Conf. on Neutron Scattering, Gatlinburg, Tenn. (1976), Conf. 760601-P1, p. 840.

[9] NyE, J. F., Propriétés Physiques des Cristaux (Dunod, Paris) 1961.

[10] VetTier, C., Thesis, Grenoble (1975). 